University of Nebraska - Lincoln

DigitalCommons@University of Nebraska - Lincoln

Faculty Publications, Department of Physics and Astronomy

Research Papers in Physics and Astronomy

$9-1-2004$

\title{
LOGNORMAL X-RAY FLUX VARIATIONS IN AN EXTREME NARROW-LINE SEYFERT 1 GALAXY
}

\author{
C. Martin Gaskell \\ University of Nebraska-Lincoln, mgaskell@ucsc.edu
}

Follow this and additional works at: https://digitalcommons.unl.edu/physicsfacpub

Part of the Physics Commons

Gaskell, C. Martin, "LOGNORMAL X-RAY FLUX VARIATIONS IN AN EXTREME NARROW-LINE SEYFERT 1 GALAXY" (2004). Faculty Publications, Department of Physics and Astronomy. 28.

https://digitalcommons.unl.edu/physicsfacpub/28

This Article is brought to you for free and open access by the Research Papers in Physics and Astronomy at DigitalCommons@University of Nebraska - Lincoln. It has been accepted for inclusion in Faculty Publications, Department of Physics and Astronomy by an authorized administrator of DigitalCommons@University of Nebraska Lincoln. 
The Astrophysical Journal, 612:L21-L24, 2004 September 1

(C) 2004. The American Astronomical Society. All rights reserved. Printed in U.S.A.

\title{
LOGNORMAL X-RAY FLUX VARIATIONS IN AN EXTREME NARROW-LINE SEYFERT 1 GALAXY
}

\author{
C. Martin Gaskell \\ Department of Physics and Astronomy, University of Nebraska, Lincoln, NE 68588-0111; mgaskell1@unl.edu \\ Received 2003 January 14; accepted 2004 July 20; published 2004 August 5
}

\begin{abstract}
We show that the large variations in the X-ray flux of the extreme narrow-line Seyfert 1 galaxy IRAS 132243809, measured during a 10 day ASCA observation, have a two-parameter lognormal distribution of multiplicative standard deviation $\sigma_{\text {mult }}=2.7$ and that the amplitude of variability at any given moment is proportional to the flux level. We find similar behavior in earlier ROSAT soft X-ray monitoring. There is no evidence of a nonvariable component. The flux-dependent behavior of the variability rules out linear shot-noise models. Although at first glance the variations of the ASCA light curve for IRAS 13224-3809 appear to exhibit nonstationary behavior with quiescent low states and more active flaring high states, our results show that the multiplicative variance is constant. Monte Carlo simulations of constant $\sigma_{\text {mult }}$ give excellent matches to the observed X-ray light curve without the need to invoke special low and high states. This supports a picture in which the long-term variability is fundamental both in active galactic nuclei and in X-ray binaries. The lognormal flux distribution and the constancy of $\sigma_{\text {mult }}$ are incompatible with the power-law distribution of flaring amplitudes expected from self-organized critical behavior. We discuss mechanisms that can generate lognormal flux distributions.
\end{abstract}

Subject headings: galaxies: active — galaxies: nuclei — galaxies: Seyfert —X-rays: galaxies

\section{INTRODUCTION}

Almost as soon as the nature of active galactic nuclei (AGNs) was recognized, it was realized that their energy output varies substantially and on surprisingly short timescales (Matthews \& Sandage 1963; Smith \& Hoffleit 1963). X-ray variability was discovered from observations made less than a decade later by the Uhuru and Copernicus satellites (Davison et al. 1975). The first detailed comparison of X-ray and optical variability (Lyutyi 1978) showed that X-ray variability, while correlated with optical variability both on long and short timescales, has a greater amplitude and shorter timescale. Because of this, soft $\mathrm{X}$-ray variability is generally considered to be the driving variability of AGNs. Despite four decades of study, however, the cause of AGN variability remains ill-understood (see, e.g., Gaskell \& Klimek 2003).

Lyutyi \& Oknyanskij (1987) made the important discovery that there was a linear relationship between the variations in the $U$-band flux $\left(\delta F_{U}\right)$ and the $U$-band flux $\left(F_{U}\right)$ for NGC 4151. This suggested that the amplitude of optical variability was directly proportional to the optical flux of the AGN. Uttley \& McHardy (2001) similarly discovered that the X-ray variability of two X-ray binaries (XRBs), the stellar mass black hole Cyg X-1 and the accreting millisecond pulsar SAX J1808.4-3658, was linearly related to the flux level (see also Uttley 2004 and Gleissner et al. 2004). They also suggested that AGNs could show a similar relationship, but they were only able to compare pairs of states of slightly differing mean luminosity in three AGNs. Vaughan et al. (2003a, 2003b) report similar relationships between variability amplitude and flux in MCG -6-3015 and Mrk 766, respectively.

If the fluctuations in a quantity are proportional to the quantity, then the distribution of values will tend to a lognormal distribution (Aitchison \& Brown 1963). Optical astronomers have usually reported AGN brightness variations in magnitudes, and these variations appear to have a normal distribution, i.e., to have a lognormal distribution in flux. However, caution is needed in interpreting optical light curves since there is always substantial contamination in the optical from the starlight of the host galaxy. In describing events in optical light curves, optical observers have commonly spoken of high events as "flares" and low events as "dropouts" or "antiflares." In contrast to optical observers, X-ray observers have almost always plotted X-ray light curves in linear units. A good example can be seen in the soft X-ray light curve of IRAS 13244-3809 (Boller et al. 1997). This appears to show flares only. In the $\mathrm{X}$-ray region there is almost no host-galaxy background to deal with, the variability is of large amplitude, and some well-sampled light curves are available.

The most extreme X-ray variability is found in so-called narrow-line Seyfert 1 galaxies (NLS1s). Narrow-line Seyfert 1 galaxies (Gaskell 1984; Osterbrock \& Pogge 1985) are so called because the central engine with its surrounding dense gas can be seen directly, as in Seyfert 1 galaxies, but the permitted optical emission lines arising from gas (the broad-line region) are much narrower than in normal Seyfert 1 galaxies. Boller et al. (1996) showed that, as a class, NLS1s also have strong soft X-ray excesses and an order-of-magnitude greater X-ray variability than would be expected for their luminosity. One of the NLS1s showing the most extreme X-ray variability to date is IRAS 13224$3809(z=0.06667)$. Boller et al. (1997) discovered X-ray variability events with amplitudes of factors of 30-50 during a 30 day monitoring with ROSAT (0.1-2.4 keV). A 1994 ASCA observation confirmed this variability (Leighly 1999). Miller et al. (2000) report the detection of large-amplitude (30\%) optical variability on a timescale of hours, but such optical variability is not common in NLS1s (Klimek et al. 2004). In this Letter we show that (1) the X-ray variability in IRAS $13224-3809$ is proportional to the mean X-ray flux level over a wide range of flux and (2) that the X-ray flux variations at different epochs and in different passbands follow a lognormal distribution.

\section{OBSERVATIONS AND ANALYSIS}

IRAS 13224-3809 was observed for 10 days in July of 2000 with the two Solid-state Imaging Spectrometers (SISs) and two Gas Imaging Spectrometers (GISs) on the ASCA satellite. The observations and analysis have been discussed in detail elsewhere (Dewangan et al. 2002). The variabilities recorded by the SIS and GIS detectors are similar, and the soft X-ray $(0.7-1.3 \mathrm{keV})$ and 


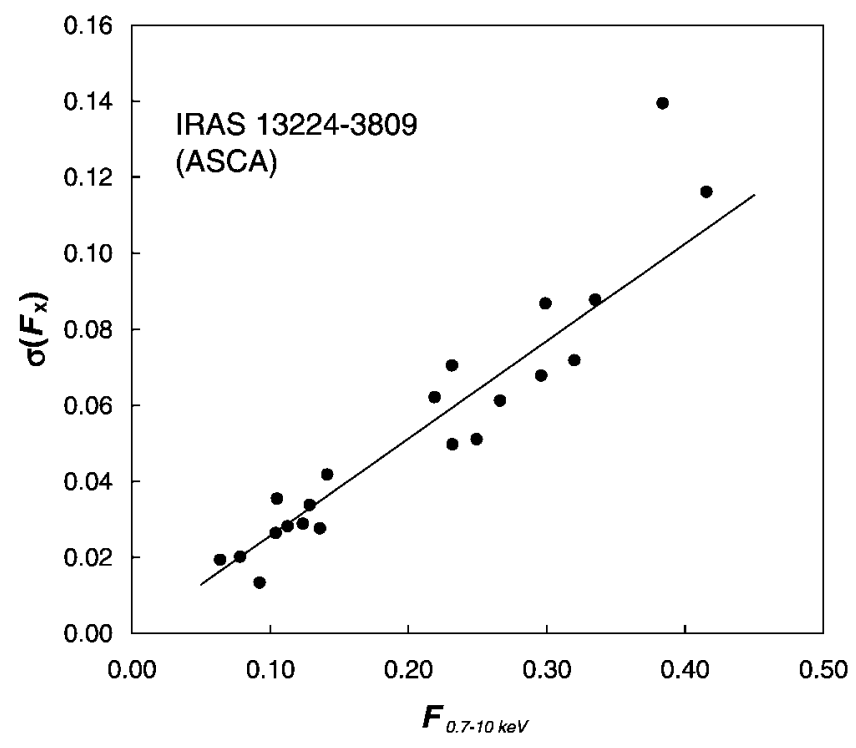

FIG. 1.-Standard deviations vs. the mean count rate in half-day bins. Both axes are in ASCA counts over the $0.7-10 \mathrm{keV}$ energy range. The fit line is $\sigma\left(F_{x}\right)=0.256 F_{x}$

hard X-ray (1.3-10 keV) variations are highly correlated (see Fig. 2 of Dewangan et al. 2002). We therefore only consider the total $0.7-10 \mathrm{keV}$ count rates as reported by Dewangan et al. During the 10 day period of observations, the peak-to-trough variations in this passband are over a factor of 30 .

The mean count rates were taken for $5 \mathrm{ks}$ intervals over the 10 day monitoring period. The means and standard deviations were then found for these mean count rates over 0.5 day ( $\sim 40 \mathrm{ks}$ ) bins. We also performed a similar analysis using the 30 day ROSAT soft X-ray data presented by Boller et al. (1997). Observational and data analysis details are as given by Boller et al.

\section{RESULTS}

In Figure 1 we show the standard deviations versus the mean count rates for the $0.7-10 \mathrm{keV} A S C A$ band. As can be seen, the line $\sigma\left(F_{x}\right)=0.26 F_{x}$ is a good fit, and hence the variability is directly proportional to the mean flux level. In Figure 2 we

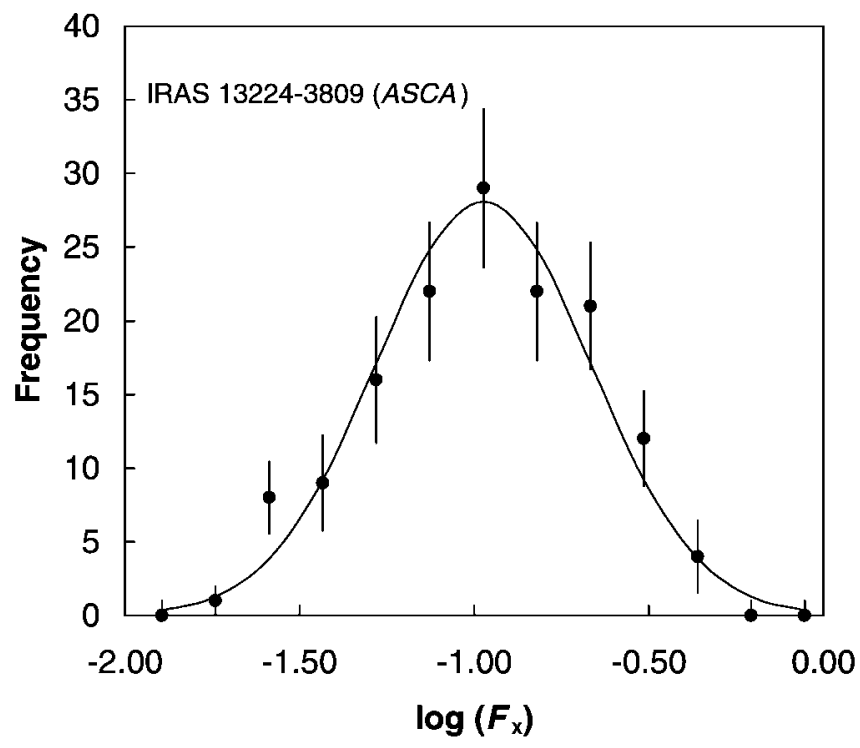

FIG. 2.-Frequency distribution of the logarithms of the ASCA count rates over $5 \mathrm{ks}$ intervals. The curve is a Gaussian of $\sigma=0.435$ dex.

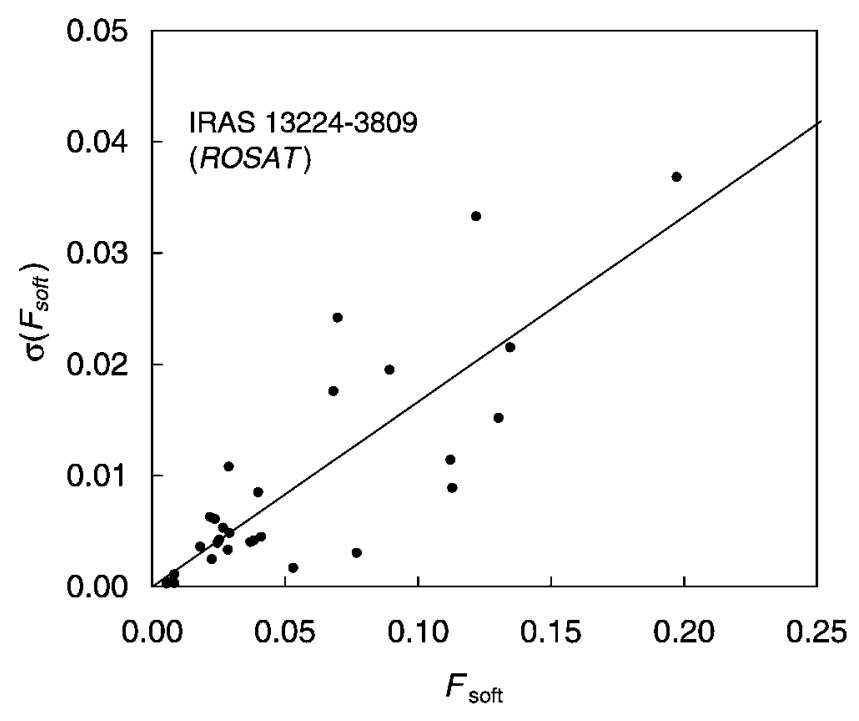

FIG. 3.- Standard deviations of the ROSAT counts vs. the mean count rate in 1 day bins. The fit line is $\sigma\left(F_{\text {soft }}\right)=0.17 F_{\text {soft }}$.

show the frequency distribution of the logarithms of the mean fluxes in the $5 \mathrm{ks}$ intervals. It can be seen that the distribution of the logarithms is well fitted by a normal distribution of $\sigma=0.43$ dex. The $\chi^{2}$ per degree of freedom is 0.86 . The distribution of $0.7-10 \mathrm{keV}$ fluxes of IRAS $13224-3809$ is therefore completely consistent with a lognormal distribution of multiplicative standard deviation $\sigma_{\text {mult }}=2.7$.

The 1996 ROSAT monitoring of Boller et al. (1997) provides a useful independent check of these results. The observations were made over 4 years earlier, with a different satellite and at a lower energy. For the ROSAT observations, the standard deviations are more uncertain than for the ASCA observations, and we had to use 1 day bins, but nonetheless it can be seen from Figure 3 that the amplitudes of the soft X-ray variations are also consistent with being proportional to the mean flux levels with $\sigma\left(F_{\text {soft }}\right) \approx 0.17 F_{\text {soft }}$. In Figure 4 it can be seen that the distribution of fluxes is again consistent with a lognormal distribution. For the ROSAT observations, the multiplicative standard deviation $\sigma_{\text {mult }}=3.5$.

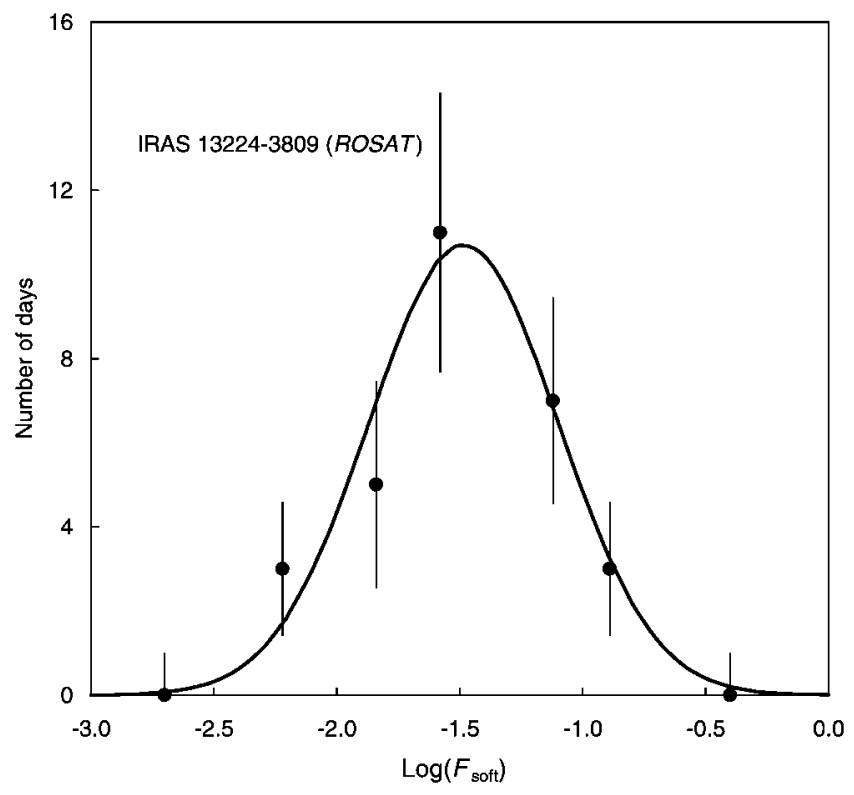

FIG. 4.-Frequency distribution of the logarithms of the ASCA count rates over 1 day intervals. The curve is a Gaussian of $\sigma=0.54$ dex. 



Fig. 5.-Five synthetic X-ray light curves and the observed ASCA light curve of IRAS 13224-3809 in 5 ks bins. See text for details. The model parameters are identical for the synthetic light curves. A fourth-order polynomial has been fitted to each light curve to show the slowly varying component.

\section{MODELLING THE X-RAY LIGHT CURVE}

It has been common to analyze X-ray light curves into relatively quiescent "low states" and more active "high states" (e.g., Dewangan et al. 2002). The results presented above suggest that there is no fundamental difference between high and low states and that a high and low state division is not significant. To illustrate this we have generated synthetic X-ray light curves with of constant $\sigma_{\text {mult }}$, and we compare these with the $2000 A S C A$ light curve. The model has only two features: a slowly varying mean flux level and fluctuations proportional to that mean flux level. We took the mean flux level to be a sine wave with a period of 10 days. The period, amplitude, phase, and constant offset were chosen to roughly match the ASCA observations. The amplitude of the lognormal fluctuations is given by $\sigma\left(F_{x}\right)=0.3 F_{x}$ to match the slope in Figure 1 . The only other parameter is the timescale of the fluctuations. This was taken to have an FWHM of $7 \mathrm{ks}$ to match the ASCA observations. We show five representative models in Figure 5, along with the actual ASCA X-ray light curve for IRAS 13224-3809. The reader might like to try to guess which is the real light curve (the answer is the middle initial of the third coauthor of the third reference in the reference list). The model parameters have been kept the same in each of the five synthetic models-only the initial seed for the random number generator is different.
Our model reproduces the following features of the real light curve:

1. As expected, the appearance is of flares rather than antiflares or dropouts.

2. The bigger flares occur when the mean flux level is higher.

3. Each light curve appears to transition fairly abruptly from a "low state" to a "high state" around bin 45, even though there is no such transition built into the model; it is merely a consequence of the gradually rising mean level.

4. The short-term variations appear (on a linear scale) to be much reduced when the AGN is at a low mean flux level (e.g., before bin 40 or after bin 130), but large "outbursts" are still possible, although rare, even at low mean flux levels (e.g., the events at bin 25 in Fig. $5 e$ and bin 130 in Fig. 5f). Note also that statistical fluctuations alone can produce apparently different levels of activity. Figure $5 f$ has the same model parameters as the other synthetic light curves. It was just generated with different random numbers.

\section{DISCUSSION}

The constancy of $\sigma_{\text {mult }}$ is similar to the linear dependence of the rms variability on X-ray flux reported by Uttley \& McHardy (2001) for Galactic accreting sources. Interestingly, the ratio 
of the standard deviation to flux is the same, $25 \%$, for the ASCA observations of IRAS 13224-3809 and for the RXTE observations of Cyg X-1 and SAX J1808.4-3658. The only significant difference is that the constant component that contributes about a quarter of the flux in the Galactic sources is lacking in IRAS 13224-3809.

Clearly the variations in IRAS $13224-3809$ are nonlinear. Vio et al. (1991) argued that the optical variations of 3C 345 are nonlinear and also nonstationary. However, in the light of the results presented above, the lognormal nature of variability needs to be considered when evaluating stationarity. There is no evidence for IRAS 13224-3809 that the multiplicative variance is not stationary.

Vio et al. (1991) further suggested that AGNs could be selforganized critical (SOC) systems; i.e., they become organized into a state where they are on the edge of instability. A pile of sand is a classic example of such an SOC system-the addition of a few grains of sand can cause a major avalanche. One of the signatures of an SOC system is that it produces power-law distributions (Bak 1996). The distribution of avalanche sizes in a sand pile, for example, follows a power-law distribution (a Pareto distribution) over many orders of magnitude. Solar flares provide an important astronomical example of this behavior (and one of potential relevance to AGNs). Dennis (1985) showed that the distribution of solar flare intensities is a power law over at least 4 orders of magnitude.

Our two main results here, that the fluctuations are proportional to the intensity and that the distribution of $\mathrm{X}$-ray intensities is lognormal, argue against AGN variations being driven by selforganized criticality. First, we have found a lognormal distribution, not the classic Pareto power-law distribution of SOC systems. Even allowing for the smoothing of the X-ray light curves that occurs both observationally, because of the need for long enough integrations to get reasonable signal-to-noise ratios, and intrinsically in the AGN, because of the finite durations of events, a power-law distribution still fails to convert into a lognormal distribution. The second argument against SOC behavior is that a power-law distribution of amplitudes of events (or a smoothing of it) also fails to produce our observed proportionality of X-ray fluctuations to the mean flux level. Although selforganized criticality may be important for some aspects of AGN behavior, the clear power-law signature seen in solar flares is lacking in the X-ray variability of IRAS 13224-3809.

Although they have received relatively little attention in astronomy so far, lognormal distributions are very common in nature. They include distributions of the critical dose of a drug, grain sizes produced by both artificial and natural means, incubation periods of diseases, time to recovery from illness, time for failure of electronic and mechanical devices, times for marriage, divorce, and death, scintillation in the Earth's atmosphere, raindrop size distributions, rainfall amounts on timescales from minutes to months, cloud sizes, pollutant concentrations, the fluctuations in many economic quantities, the abundance of biological species, velocities in air, the time to solve a research problem, the lengths of telephone calls, and the distribution of word lengths in this Letter (see Aitchison \& Brown 1963, Johnson \& Kotz 1970, and Crow \& Shimizu 1988). Many of these diverse occurrences of lognormal distributions can be attributed to four closely related geneses:

1. If an outcome is the result of a product of various factors, then the multiplicative central-limit theorem says that the distribution of outcomes tends to a lognormal distribution.

2. If the change in a variate obeys the law of proportionate effect, in that the change at any step in the process is a random proportion of the previous value, then the variate will tend to be lognormally distributed. This has application to growth processes and mergings.

3. Comminutive processes (e.g., crushing rocks by artificial or natural processes) produce lognormal distributions of sizes.

4. If the rate of change of a variate is proportional to the variate times a rate constant and if this rate constant is normally distributed, then at a future time the variate will have a lognormal distribution.

A lognormal distribution of X-ray fluxes suggests that the emitting regions could have a lognormal size distribution or that the energies could have a lognormal distribution. Potentially any of the generating mechanisms could produce the required driving lognormal distributions.

Whatever the causes, clearly both the flux-dependent variability and the lognormal flux distributions are fundamental factors that any model of AGN variability must explain. The flux-dependent variability of both AGNs and XRBs is very important because it implies similarities in the underlying causes of the variability.

I am grateful to Phil Uttley and the anonymous referee for useful comments. This research was supported in part by NASA grant NAG5-6506 and NSF grant AST 03-07912.

\section{REFERENCES}

Aitchison, J., \& Brown, J. A. C. 1963, The Lognormal Distribution (Cambridge: Cambridge Univ. Press)

Bak, P. 1996, How Nature Works: The Science of Self-Organized Criticality (New York: Springer)

Boller, Th., Brandt, W. N., Fabian, A. C., \& Fink, H. H. 1997, MNRAS, 289, 393

Boller, Th., Brandt, W. N., \& Fink 1996, A\&A, 305, 53

Crow, E. L., \& Shimizu, K. 1988, Lognormal Distributions: Theory and Applications (New York: Dekker)

Davison, J. P. N., Culhane, J. L., Mitchell, R. J., \& Fabian, A. C. 1975, ApJ, 196, L23

Dennis, B. R. 1985, Sol. Phys., 100, 465

Dewangan, G. C., Boller, Th., Sing, K. P., \& Leighly, K. M. 2002, A\&A, 390, 65

Gaskell, C. M. 1984, Astrophys. Lett., 24, 43

Gaskell, C. M., \& Klimek, E. S. 2003, Astron. Astrophys. Trans., 22, 661

Gleissner, T., Wilms, J., Pottschmidt, K., Uttley, P., Nowak, M. A., \& Staubert, R. 2004, A\&A, 414, 1091
Johnson, N. L., \& Kotz, S. 1970, Continuous Univariate Distributions: Distributions in Statistics (New York: Houghton Mifflin)

Klimek, E. S., Gaskell, C. M., \& Hedrick, C. H. 2004, ApJ, 609, 69

Leighly, K. M. 1999, ApJS, 125, 297

Lyutyi, V. M. 1978, Soviet Astron. Lett., 4, 267

Lyutyi, V. M., \& Oknyanskij, V. L. 1987, AZh, 64, 465

Matthews, T. A., \& Sandage, A. R. 1963, ApJ, 138, 30

Miller, H. R., Ferrara, E. C., McFarland, J. P., Wilson, J. W., Daya, A. B., \& Fried, R. E. 2000, NewA Rev., 44, 539

Osterbrock, D. E., \& Pogge, R. W. 1985, ApJ, 297, 166

Smith, H. J., \& Hoffleit, D. 1963, Nature, 198, 650

Uttley, P. 2004, MNRAS, 347, L61

Uttley, P., \& McHardy, I. M. 2001, MNRAS, 323, L26

Vaughan, S., Edelson, R., Warwick, R. S., \& Uttley, P. 2003a, MNRAS, 345, 1271

Vaughan, S., Fabian, A. C., \& Nandra, K. 2003b, MNRAS, 339, 1237

Vio, R., Cristiani S., Lessi, O., \& Salvadori, L. 1991, ApJ, 380, 351 